\title{
TWO CLOSED GEODESICS ON COMPACT SIMPLY CONNECTED BUMPY FINSLER MANIFOLDS
}

\author{
Huagui Duan, Yiming Long \& Wei Wang
}

\begin{abstract}
We prove the existence of at least two distinct closed geodesics on a compact simply connected manifold $M$ with a bumpy and irreversible Finsler metric, when $H^{*}(M ; \mathbf{Q}) \cong T_{d, h+1}(x)$ for some integer $h \geq 2$ and even integer $d \geq 2$. Consequently, together with earlier results on $S^{n}$, it implies the existence of at least two distinct closed geodesics on every compact simply connected manifold $M$ with a bumpy irreversible Finsler metric.
\end{abstract}

\section{Introduction and main results}

A famous long-standing conjecture in Riemannian geometry that claims the existence of infinitely many distinct closed geodesics on every compact Riemannian manifold is still open, although it has been proved for many cases. It was quite surprising when A. Katok [Kat] in 1973 constructed irreversible Finsler metrics on spheres with only finitely many closed geodesics and that all these closed geodesics were non-degenerate. This paper is devoted to finding a lower bound on the number of closed geodesics on compact simply connected bumpy Finsler manifolds.

A closed curve on a Finsler manifold is a closed geodesic if it is locally the shortest path connecting any two nearby points on this curve (cf. [She]). As usual, on a Finsler manifold $(M, F)$, the $m$ th iterate $c^{m}$ of a closed curve $c: S^{1}=\mathbf{R} / \mathbf{Z} \rightarrow M$ is defined by $c^{m}(t)=c(m t)$. A closed geodesic $c$ is prime if it is not a multiple covering (i.e., iterate) of any other closed geodesics. The inverse curve $c^{-1}$ of $c$ is defined by $c^{-1}(t)=c(1-t)$ for $t \in \mathbf{R}$. Note that unlike the case of a Riemannian manifold, the inverse curve $c^{-1}$ of a closed geodesic $c$ on a irreversible Finsler manifold needs not be a geodesic. We call two prime closed geodesics $c$ and $d$ distinct if there is no $\theta \in(0,1)$ such that $c(t)=d(t+\theta)$ for all $t \in \mathbf{R}$. We shall omit the word distinct when we talk about more than one prime closed geodesic. On a reversible Finsler (or Riemannian) manifold, two closed geodesics $c$ and $d$ are called geometrically distinct if $c\left(S^{1}\right) \neq d\left(S^{1}\right)$ in $M$. For a closed geodesic $c$ on $(M, F)$ with $n=\operatorname{dim} M$,

Received 12/4/2014. 
denote by $P_{c}$ the linearized Poincaré map of $c$. Then $P_{c} \in \operatorname{Sp}(2 n-2)$ is symplectic. A closed geodesic $c$ is called non-degenerate if 1 is not an eigenvalue of $P_{c}$. A Finsler manifold $(M, F)$ is called bumpy if all the closed geodesics on $M$ are non-degenerate. Note that bumpy Finsler metrics are generic in the set of Finsler metrics.

For surveys on closed geodesics on Riemannian and Finsler manifolds, we refer to Bangert [Ban] and Long [Lon4] (see also [Ano]). Note that by the classical theorem of Lyusternik and Fet $[\mathbf{L y F}]$ in 1951, there exists at least one closed geodesic on every compact Riemannian manifold. Their proof is variational and works also for the Finsler manifolds. Later proofs of this existence result can be found in [Ban] in German as well as [Kli] and [Jos] in English. Besides other papers, in [Rad1], [Rad2], and [Rad3], Rademacher studied the existence and stability of closed geodesics on Finsler manifolds. Bangert and Long proved in 2005 the existence of at least two distinct closed geodesics on every Finsler 2-sphere $\left(S^{2}, F\right)$, which was published in 2010 [BaL] (cf. [Lon4] from 2006). Then in Duan and Long [DuL1] and Rademacher [Rad4], the existence of at least two distinct closed geodesics on every bumpy $n$-sphere was proved independently. In [Rad5], Rademacher further proved there exist two prime closed geodesics on any $\mathbf{C P}^{\mathbf{2}}$ with a bumpy irreversible Finsler metric. Related more recent results can be found in $[\mathbf{L o W}]$, [Wan], and [HiR].

Note that for compact simply connected bumpy reversible Finsler manifolds, Fet proved [Fet] in 1965 the existence of at least two distinct closed geodesics. Our aim in this paper is to prove such a theorem for these manifolds with bumpy irreversible Finsler metrics.

Theorem 1.1. There exist at least two closed geodesics on every compact simply connected manifold $M$ with a bumpy irreversible Finsler metric $F$ satisfying $H^{*}(M ; \mathbf{Q}) \cong T_{d, h+1}(x)$ for some integer $h \geq 2$ and even integer $d \geq 2$.

For a compact simply connected Finsler manifold $(M, F)$, if the main theorem in $[\mathbf{G r M}]$ is applicable (i.e., the Betti number sequence of the free loop space $\Lambda M$ of $M$ is unbounded) then there exist infinitely many distinct closed geodesics on $M$. If this sequence is bounded, then $H^{*}(M ; \mathbf{Q}) \cong T_{d, h+1}(x)$ holds for some integers $h \geq 1$ and $d \geq 2$ by [ViS]. In this case, when $d$ is odd, then $x^{2}=0$ and $h=1$ in $T_{d, h+1}(x)$. Thus $M$ is rationally homotopy equivalent to $S^{d}$ (see [Rad1, Remark 2.5] and [Hin]). Note also that $M$ is rationally homotopic to $S^{d}$ for all $d \geq 2$ when $h=1$.

Notice that the monotonicity of iterated Morse indices of closed geodesics has played an important and crucial role in some works on closed geodesic problems (see $[\mathbf{D u L 1}],[\mathbf{R a d} 4]$, and $[\mathbf{W a n}]$ ). For example, Rademacher [Rad4] and Wang [Wan] have used the monotonicity and 
the common index jump theorem obtained in $[\mathbf{L o Z}]$ to get some multiplicity results on Finsler spheres.

However, the monotonicity may no longer hold in general because of smaller initial Morse index $i(c)$ (see Lemma 3.1, below) when one considers closed geodesics on a compact simply connected manifold $M$ with $H^{*}(M ; \mathbf{Q}) \cong T_{d, h+1}(x)$ for some integer $h \geq 2$ and even integer $d \geq 2$. In this paper, the main novelty is to deal with the difficulty of lack of index growth monotonicity.

Indeed, in the proof of Theorem 1.1, we assume that there exists only one prime closed geodesic on such a manifold. On one hand, if a compact simply connected manifold has a bumpy metric and only one closed geodesic, the energy is a perfect equivariant Morse function, and the homology has one generator for each $\mathrm{SO}(2)$ orbit of closed geodesics that satisfies an orientability condition. Moreover, the dimensions in which non-zero homology appears all have the same parity. So the Morse inequalities become the equalities (see Lemma 3.2, below). This idea has been used more or less in $[\mathbf{D u L 1}],[\operatorname{Rad} 4],[\operatorname{Rad} 5]$, and $[\mathbf{W a n}]$. On the other hand, in order to investigate the question of whether or not it is possible for the index of the iterates of a single closed geodesic to occur in the sequence of the free loop space, we use the quasi-monotonicity of index growth for closed geodesics proved in [DuL2] to truncate the Morse series, and use the mean index equality of Rademacher to get a contradiction to the Morse inequality. Such ideas are used by the first two authors in their recent study (see, for example, [LoD] and [DuL2]) on the multiplicity of closed geodesics, and the current paper can be viewed as a simplified model of such a study that turns out to be perfect.

Now, together with results in [DuL1] and $[\mathbf{R a d} 4]$, Theorem 1.1 yields the following corollary.

Corollary 1.2. There exist always at least two closed geodesics on every compact simply connected bumpy irreversible Finsler manifold $(M, F)$.

Remark 1.3. When one considers any closed geodesic $c$ on a compact manifold with a reversible Finsler metric, its inverse curve $c^{-1}$ has the same energy and plays the same role in the variational setting of the energy functional $E$ on $\Lambda M$ as $c$. Specially, the $m$ th iterates $c^{m}$ and $c^{-m}$ of a closed geodesic $c$ and its inverse curve $c^{-1}$ have precisely the same Morse indices, nullities, and critical modules. When the metric is bumpy, the Morse-type number sequence and Betti number sequence are lacunary, and thus no cancelation in homologies. Thus if we assume that there exists only one prime closed geodesic on a compact simply connected bumpy reversible Finsler manifold, then all Morse-type numbers defined in (3.1), below, are even. But the first non-zero Betti number $b_{d-1}$ is 1 (cf. [Rad1, Theorem 2.4 and Remark 2.5] and [DuL2, Lemma 
2.5]). This yields a contradiction to the Morse theory (cf. Lemma 3.2 below). This gives a different and simpler proof of Fet's theorem [Fet], i.e., Corollary 1.2 holds in the case of such reversible Finsler manifolds too.

In this paper, let $\mathbf{N}, \mathbf{N}_{0}, \mathbf{Z}, \mathbf{Q}$, and $\mathbf{R}$ denote the sets of natural integers, non-negative integers, integers, rational numbers, and real numbers respectively. We use only singular homology modules with Qcoefficients. We use also notations $[a]=\max \{k \in \mathbf{Z} \mid k \leq a\}, \mathcal{E}(a)=$ $\min \{k \in \mathbf{Z} \mid k \geq a\}, \varphi(a)=\mathcal{E}(a)-[a]$, and $\{a\}=a-[a]$ for any $a \in \mathbf{R}$.

Acknowledgments. The first author is partially supported by NSFC (Nos.11131004, 11471169), LPMC of MOE of China, and Nankai University. The second author is partially supported by NSFC (No.11131004), MCME and LPMC of MOE of China, Nankai University, and BCMIIS of Capital Normal University. The third author is partially supported by NSFC (Nos. 11222105, 11431001), Foundation for the Author of National Excellent Doctoral Dissertation of PR China No. 201017. The authors sincerely thank the referees for their careful reading, valuable comments, and suggestions on this paper.

\section{Preliminary results on closed geodesics}

2.1. Critical modules of iterations of closed geodesics. Let $M=$ $(M, F)$ be a compact Finsler manifold. The space $\Lambda=\Lambda M$ of $H^{1}$-maps $\gamma: S^{1} \rightarrow M$ has a natural structure of Riemannian Hilbert manifolds on which the group $S^{1}=\mathbf{R} / \mathbf{Z}$ acts continuously by isometries. This action is defined by $(s \cdot \gamma)(t)=\gamma(t+s)$ for all $\gamma \in \Lambda$ and $s, t \in S^{1}$. For any $\gamma \in \Lambda$, the energy functional is defined by

$$
E(\gamma)=\frac{1}{2} \int_{S^{1}} F(\gamma(t), \dot{\gamma}(t))^{2} d t
$$

It is $C^{1,1}$ and invariant under the $S^{1}$-action. The critical points of $E$ of positive energies are precisely the closed geodesics $\gamma: S^{1} \rightarrow M$. The index form of the functional $E$ is well defined along any closed geodesic $c$ on $M$, which we denote by $E^{\prime \prime}(c)$. As usual, we denote by $i(c)$ and $\nu(c)$ the Morse index and nullity of $E$ at $c$. In the text below, we use the following notation:

$$
\Lambda^{\kappa}=\{d \in \Lambda \mid E(d) \leq \kappa\}, \Lambda^{\kappa-}=\{d \in \Lambda \mid E(d)<\kappa\}, \forall \kappa \geq 0 .
$$

For a closed geodesic $c$, we set $\Lambda(c)=\{\gamma \in \Lambda \mid E(\gamma)<E(c)\}$.

For a closed geodesic $c$, the mean index $\hat{i}(c)$ is defined as usual by $\hat{i}(c)=\lim _{m \rightarrow \infty} i\left(c^{m}\right) / m$. Using singular homology with rational coefficients, we consider the following critical $\mathbf{Q}$-module of a closed geodesic $c \in \Lambda$ :

$$
\bar{C}_{*}(E, c)=H_{*}\left(\left(\Lambda(c) \cup S^{1} \cdot c\right) / S^{1}, \Lambda(c) / S^{1}\right) .
$$


Proposition 2.1. (cf. [Rad2, Satz 6.11]) Let $c$ be a prime closed geodesic on a bumpy Finsler manifold $(M, F)$. Then there holds

$$
\bar{C}_{q}\left(E, c^{m}\right)= \begin{cases}\mathbf{Q}, & \text { if } i\left(c^{m}\right)-i(c) \in 2 \mathbf{Z} \text { and } q=i\left(c^{m}\right), \\ 0, & \text { otherwise. }\end{cases}
$$

Definition 2.2. (cf. [Rad1, Definition 1.6]) For a closed geodesic $c$, let $\gamma_{c} \in\left\{ \pm \frac{1}{2}, \pm 1\right\}$ be the invariant defined by $\gamma_{c}>0$ if and only if $i(c)$ is even, and $\left|\gamma_{c}\right|=1$ if and only if $i\left(c^{2}\right)-i(c)$ is even.

Proposition 2.3. (cf. [Rad1, Theorem 3.1] and [Rad2, Satz 7.9]) Let $(M, F)$ be a compact simply connected bumpy Finsler manifold with $H^{*}(M, \mathbf{Q})=T_{d, h+1}(x)$. Denote prime closed geodesics on $(M, F)$ with positive mean indices by $\left\{c_{j}\right\}_{1 \leq j \leq q}$ for some $q \in \mathbf{N}$. Then the identity

$$
\sum_{j=1}^{q} \frac{\gamma_{c_{j}}}{\hat{i}\left(c_{j}\right)}=B(d, h)= \begin{cases}-\frac{h(h+1) d}{2 d(h+1)-4}, & d \text { is even }, \\ \frac{d+1}{2 d-2}, & d \text { is odd }\end{cases}
$$

holds, where $\operatorname{dim} M=h d, h=1$ when $M$ is a sphere $S^{d}$ of dimension $d$.

2.2. The structure of $H_{*}\left(\Lambda M / S^{1}, \Lambda^{0} M / S^{1} ; \mathbf{Q}\right)$. Set $\bar{\Lambda}^{0}=\bar{\Lambda}^{0} M=$ $\{$ constant point curves in $M\} \cong M$. Let $(X, Y)$ be a space pair such that the Betti numbers $b_{i}=b_{i}(X, Y)=\operatorname{dim} H_{i}(X, Y ; \mathbf{Q})$ are finite for all $i \in \mathbf{Z}$. As usual, the Poincaré series of $(X, Y)$ is defined by the formal power series $P(X, Y)=\sum_{i=0}^{\infty} b_{i} t^{i}$. As discussed before Corollary 1.2, in the following we consider only the case when $d \geq 2$ is even and $h \geq 2$.

Lemma 2.4. (cf. [Rad1, Theorem 2.4] and [DuL2, Lemma 2.6]) Let $M$ be a compact simply connected manifold with $H^{*}(M ; \mathbf{Q}) \cong T_{d, h+1}(x)$ for some integer $h \geq 2$ and even integer $d \geq 2$. Let $D=d(h+1)-2$ and

$\Omega(d, h)=\{k \in 2 \mathbf{N}-1 \quad \mid \quad i D \leq k-(d-1)=i D+j d \leq i D+(h-1) d$ for some $i \in \mathbf{N}$ and $j \in[1, h-1]\}$.

Then the Betti numbers of the free loop space of $M$ defined by $b_{q}=$ $\operatorname{rank} H_{q}\left(\Lambda M / S^{1}, \Lambda^{0} M / S^{1} ; \mathbf{Q}\right)$ for $q \in \mathbf{Z}$ are given by

$$
b_{q}= \begin{cases}0, & \text { if } q \text { is even or } q \leq d-2, \\ {\left[\frac{q-(d-1)}{d}\right]+1,} & \text { if } q \in 2 \mathbf{N}-1 \\ h+1, & \text { and } d-1 \leq q<d-1+(h-1) d, \\ h, & \text { if } q \in \Omega(d, h), \\ \text { otherwise. }\end{cases}
$$

In particular, for every integer $l \geq d-1+(h-1) d=h d-1$, we have

$$
\sum_{q=0}^{l} b_{q}<h\left(\frac{D}{2}+1\right) \frac{l-(d-1)}{D}-\frac{h(h-1) d}{4}+2 .
$$


2.3. Morse indices of closed geodesics. In [Lon1], from 1999, Long established the basic normal form decomposition of symplectic matrices. Based on this result, he further established the precise iteration formulae of indices of symplectic paths in [Lon2], from 2000.

As in [Lon2], we denote the following:

$$
\begin{aligned}
(2.8) N_{1}(\lambda, a) & =\left(\begin{array}{cc}
\lambda & a \\
0 & \lambda
\end{array}\right), \quad \text { for } \lambda= \pm 1, a \in \mathbf{R}, \\
(2.9) \quad H(b) & =\left(\begin{array}{cc}
b & 0 \\
0 & b^{-1}
\end{array}\right), \quad \text { for } b \in \mathbf{R} \backslash\{0, \pm 1\}, \\
(2.10) \quad R(\theta) & =\left(\begin{array}{cc}
\cos \theta & -\sin \theta \\
\sin \theta & \cos \theta
\end{array}\right), \quad \text { for } \theta \in(0, \pi) \cup(\pi, 2 \pi), \\
N_{2}\left(e^{\theta \sqrt{-1}}, B\right)= & \left(\begin{array}{cc}
R(\theta) & B \\
0 & R(\theta)
\end{array}\right), \quad \text { for } \theta \in(0, \pi) \cup(\pi, 2 \pi) \text { and } \\
(2.11) & B=\left(\begin{array}{cc}
b_{1} & b_{2} \\
b_{3} & b_{4}
\end{array}\right) \text { with } b_{j} \in \mathbf{R}, \quad \text { and } b_{2} \neq b_{3} .
\end{aligned}
$$

Here $N_{2}\left(e^{\theta \sqrt{-1}}, B\right)$ is non-trivial if $\left(b_{2}-b_{3}\right) \sin \theta<0$, and trivial if $\left(b_{2}-b_{3}\right) \sin \theta>0$.

As in [Lon2], the $\diamond$-sum (direct sum) of any two real matrices is defined by

$$
\left(\begin{array}{cc}
A_{1} & B_{1} \\
C_{1} & D_{1}
\end{array}\right)_{2 i \times 2 i} \diamond\left(\begin{array}{cc}
A_{2} & B_{2} \\
C_{2} & D_{2}
\end{array}\right)_{2 j \times 2 j}=\left(\begin{array}{cccc}
A_{1} & 0 & B_{1} & 0 \\
0 & A_{2} & 0 & B_{2} \\
C_{1} & 0 & D_{1} & 0 \\
0 & C_{2} & 0 & D_{2}
\end{array}\right) .
$$

For every $P \in \operatorname{Sp}(2 d)$, the homotopy set $\Omega(P)$ of $P$ in $\operatorname{Sp}(2 d)$ is defined by

$$
\begin{aligned}
\Omega(P)=\{N \in \operatorname{Sp}(2 d) \mid \sigma(N) \cap \mathbf{U}= & \sigma(P) \cap \mathbf{U} \equiv \Gamma, \\
& \text { and } \left.\nu_{\omega}(N)=\nu_{\omega}(P), \quad \forall \omega \in \Gamma\right\},
\end{aligned}
$$

where $\sigma(P)$ denotes the spectrum of $P, \nu_{\omega}(P) \equiv \operatorname{dim}_{\mathbf{C}} \operatorname{ker}_{\mathbf{C}}(P-\omega I)$ for $\omega \in \mathbf{U}$. The homotopy component $\Omega^{0}(P)$ of $P$ in $\operatorname{Sp}(2 d)$ is defined by the path-connected component of $\Omega(P)$ containing $P$. Then the following decomposition theorem is proved in $[$ Lon1] and [Lon2].

Theorem 2.5. (cf. [Lon1, Theorem 7.8], [Lon2, Theorems 1.2 and 1.3]; see also [Lon3, Theorem 1.8.10, Lemma 2.3.5, Theorem 8.3.1]) For every $P \in \operatorname{Sp}(2 n)$, there exists a continuous path $f \in \Omega^{0}(P)$ such that 
$f(0)=P$ and

$$
\begin{aligned}
f(1)= & N_{1}(1,1)^{\diamond p_{-}} \diamond I_{2 p_{0}} \diamond N_{1}(1,-1)^{\diamond p_{+}} \\
& \diamond N_{1}(-1,1)^{\diamond q_{-}} \diamond\left(-I_{2 q_{0}}\right) \diamond N_{1}(-1,-1)^{\diamond q_{+}} \\
& \diamond N_{2}\left(e^{\alpha_{1} \sqrt{-1}}, A_{1}\right) \diamond \cdots \diamond N_{2}\left(e^{\alpha_{r_{*}} \sqrt{-1}}, A_{r_{*}}\right) \\
& \diamond N_{2}\left(e^{\beta_{1} \sqrt{-1}}, B_{1}\right) \diamond \cdots \diamond N_{2}\left(e^{\beta_{r_{0}} \sqrt{-1}}, B_{r_{0}}\right) \\
& \diamond R\left(\theta_{1}\right) \diamond \cdots \diamond R\left(\theta_{k}\right) \diamond R\left(\theta_{k+1}\right) \diamond \cdots \diamond R\left(\theta_{r}\right) \diamond H(2)^{\diamond h},
\end{aligned}
$$

where $\frac{\theta_{j}}{2 \pi} \notin \mathbf{Q} \cap(0,1)$ for $1 \leq j \leq k$ and $\frac{\theta_{j}}{2 \pi} \in \mathbf{Q} \cap(0,1)$ for $k+1 \leq j \leq r$; $N_{2}\left(e^{\alpha_{j} \sqrt{-1}}, A_{j}\right)$ 's are non-trivial and $N_{2}\left(e^{\beta_{j} \sqrt{-1}}, B_{j}\right)$ 's are trivial; and non-negative integers $p_{-}, p_{0}, p_{+}, q_{-}, q_{0}, q_{+}, r, r_{*}, r_{0}, h$ satisfy

$$
p_{-}+p_{0}+p_{+}+q_{-}+q_{0}+q_{+}+r+2 r_{*}+2 r_{0}+h=n .
$$

Let $\gamma \in \mathcal{P}_{\tau}(2 n)=\{\gamma \in C([0, \tau], \operatorname{Sp}(2 n)) \mid \gamma(0)=I\}$. Denote the basic normal form decomposition of $P \equiv \gamma(\tau)$ by (2.13). Then we have

$$
\begin{aligned}
& i\left(\gamma^{m}\right)= m\left(i(\gamma)+p_{-}+p_{0}-r\right)+2 \sum_{j=1}^{r} \mathcal{E}\left(\frac{m \theta_{j}}{2 \pi}\right)-r \\
&(2.14) \quad-p_{-}-p_{0}-\frac{1+(-1)^{m}}{2}\left(q_{0}+q_{+}\right)+2 \sum_{j=1}^{r_{*}} \varphi\left(\frac{m \alpha_{j}}{2 \pi}\right)-2 r_{*} .
\end{aligned}
$$

By $[\mathbf{L i u}]$ and $[\mathbf{L i L}]$, these results can be applied to closed geodesic problems.

Theorem 2.6. (cf. [DuL2, Theorem 3.21, Corollary 3.24]) Let c be a orientable closed geodesic with mean index $\hat{i}(c)>0$ on a bumpy Finsler manifold $(M, F)$. Denote the basic normal form decomposition of the linearized Poincaré map $P_{c}$ of $c$ by (2.13). Then there exist an integer $A$ with $[(k+1) / 2] \leq A \leq k$ and a subset $P$ of integers $\{1, \ldots, k\}$ with $A$ integers such that for any $\epsilon \in(0,1 / 4)$ there exists an arbitrarily large even integer $T$ that can be chosen to be the multiple of any fixed integer satisfying

$$
\begin{aligned}
& \left\{\frac{T \theta_{j}}{2 \pi}\right\}>1-\epsilon, \quad \text { for } j \in P, \\
& \left\{\frac{T \theta_{j}}{2 \pi}\right\}<\epsilon, \quad \text { for } j \in\{1, \ldots, k\} \backslash P .
\end{aligned}
$$

Consequently, we have

$$
\begin{aligned}
& (2.17) \quad i\left(c^{m}\right) \geq i\left(c^{T}\right)+i(c)+(2 A-k), \quad \forall m \geq T+1, \\
& (2.18) \quad i\left(c^{m}\right) \leq i\left(c^{T}\right)-i(c)+(2 A-k), \quad \forall 1 \leq m \leq T-1 .
\end{aligned}
$$




\section{Proof of main theorem}

By the discussion before Corollary 1.2 in Section 1, we consider only the multiplicity problem on a compact simply connected bumpy Finsler manifold $(M, F)$ satisfying $H^{*}(M ; \mathbf{Q}) \cong T_{d, h+1}(x)$ for $h \geq 2$ and even $d \geq 2$. We shall prove Theorem 1.1 by contradiction. Thus we assume the following condition holds:

(OSCG). there exists only one prime closed geodesic $c$ on the manifold $(M, F)$.

Note that by the Morse theory and (2.6) of Lemma 2.4, in order to generate the non-trivial Betti number $b_{d-1}=1$, the initial Morse index of this closed geodesic $c$ must be $d-1$. That is,

Lemma 3.1. Under the condition (OSCG), there holds $i(c)=d-1 \in$ $2 \mathbf{N}-1$.

Now under the condition (OSCG), we define

$$
m_{q}=\#\left\{m \geq 1 \mid \bar{C}_{q}\left(E, c^{m}\right) \neq 0\right\}, \quad \forall q \in \mathbf{Z} .
$$

Note that since $(M, F)$ is bumpy, by Proposition 2.1 we have $i\left(c^{m}\right)=q$ whenever $\bar{C}_{q}\left(E, c^{m}\right) \neq 0$.

Then we have the following lemma.

Lemma 3.2. Under the condition (OSCG), there hold

$$
m_{2 q+1}=b_{2 q+1}, \quad m_{2 q}=b_{2 q}=0, \quad \forall q \in \mathbf{Z} .
$$

Proof. Under the condition (OSCG), as usual we define the Morsetype numbers by

$$
M_{q}=\sum_{m \geq 1} \operatorname{dim} \bar{C}_{q}\left(E, c^{m}\right), \quad \forall q \in \mathbf{N}_{0} .
$$

By Proposition 2.1, we have $\operatorname{dim} \bar{C}_{q}\left(E, c^{m}\right)=1$ when $\bar{C}_{q}\left(E, c^{m}\right) \neq 0$. Therefore, $M_{q}=m_{q}$ holds for all $q \in \mathbf{Z}$.

By Lemma 3.1, we have $i(c)=d-1 \in 2 \mathbf{N}-1$. Thus $\bar{C}_{q}\left(E, c^{m}\right)=0$ when $i\left(c^{m}\right)$ is even by Proposition 2.1. This implies $m_{2 q}=0$ for all $q \in \mathbf{N}_{0}$. Then, by (2.6) of Lemma 2.4, it yields $M_{2 q}=b_{2 q}=0$ for all $q \in \mathbf{N}_{0}$. Then (3.2) obviously follows from the Morse inequalities (3.3) $M_{q}-M_{q-1}+\cdots+(-1)^{q} M_{0} \geq b_{q}-b_{q-1}+\cdots+(-1)^{q} b_{0}, \forall q \in \mathbf{N}_{0}$, and the proof is complete. q.e.d.

Proof of Theorem 1.1. Since the Finsler metric $F$ is bumpy, the decomposition in Theorem 2.5 of the linearized Poincaré map has the following form:

$$
\begin{aligned}
f_{c}(1)= & R\left(\theta_{1}\right) \diamond \cdots \diamond R\left(\theta_{k}\right) \diamond H(2)^{\diamond h} \\
& \diamond N_{2}\left(e^{\alpha_{1} \sqrt{-1}}, A_{1}\right) \diamond \cdots \diamond N_{2}\left(e^{\alpha_{k_{*}} \sqrt{-1}}, A_{k_{*}}\right) \\
& \diamond N_{2}\left(e^{\beta_{1} \sqrt{-1}}, B_{1}\right) \diamond \cdots \diamond N_{2}\left(e^{\beta_{k_{0}} \sqrt{-1}}, B_{k_{0}}\right),
\end{aligned}
$$


where $\frac{\theta_{j}}{2 \pi} \notin \mathbf{Q}$ for $1 \leq j \leq k ; \frac{\alpha_{j}}{2 \pi} \notin \mathbf{Q}$ for $1 \leq j \leq k_{*} ; \frac{\beta_{j}}{2 \pi} \notin \mathbf{Q}$ for $1 \leq j \leq k_{0}$ and

$$
k+h+2 k_{*}+2 k_{0}=d h-1,
$$

where we have used the fact $p_{-}=p_{0}=q_{0}=q_{+}=0$ in (2.14). Using the bumpy assumption, Theorems 8.2.3 and 8.2.4 of [Lon3], and Lemma 3.1 , as further studied in Section 4 of [DuL1], we then obtain

$$
i\left(c^{m}\right)=m(d-1-k)+2 \sum_{j=1}^{k}\left[\frac{m \theta_{j}}{2 \pi}\right]+k, \quad \nu\left(c^{m}\right)=0, \quad \forall m \geq 1,
$$

where we used the fact that $\varphi(a)=1$ if $a \notin \mathbf{Z}$.

Next, we carry out our proofs in three cases according to the parity of $k$ and the case of $k=0$.

Case 1. $k=d h-1-\left(h_{-}+h_{+}+2 k_{*}+2 k_{0}\right) \in 2 \mathbf{N}-1$.

In this case, $i\left(c^{m}\right)$ is odd and $i\left(c^{m}\right)-i(c)$ is even for any $m \in \mathbf{N}$ by (3.5). Thus by Definition 2.2 we get $\gamma_{c}=-1$, which, together with Proposition 2.3, yields

$$
-\frac{1}{\hat{i}(c)}=B(d, h)=-\frac{h(h+1) d}{2 d(h+1)-4} .
$$

Thus by (3.5) we obtain

$$
d-1-k+\sum_{i=1}^{k} \frac{\theta_{i}}{\pi}=\hat{i}(c)=\frac{2 d(h+1)-4}{h(h+1) d} .
$$

So in this case, we have

$$
\begin{aligned}
i(c) & =d-1, \\
m \hat{i}(c) & =(d-1-k) m+2 \sum_{i=1}^{k} \frac{m \theta_{i}}{2 \pi} \\
& =\frac{2(d h+d-2) m}{h(h+1) d}, \quad \forall m \geq 1 .
\end{aligned}
$$

Claim 1. There exist an integer $A$ with $\left[\frac{k+1}{2}\right] \leq A \leq k$ and a subset $P$ of integers $\{1, \ldots, k\}$ with $A$ integers such that there exist infinitely many arbitrarily large integers $T \in h(h+1) d \mathbf{N}$ satisfying

$$
i\left(c^{T}\right)=\frac{2(d h+d-2) T}{h(h+1) d}-(2 A-k) .
$$

In fact, by Theorem 2.6 (cf. [DuL2, Corollary 3.19]), there exist an integer $A$ with $\left[\frac{k+1}{2}\right] \leq A \leq k$ and a subset $P$ of integers $\{1, \ldots, k\}$ 
with $A$ integers such that for any $\epsilon \in\left(0, \frac{1}{2 k}\right)$ there exist infinitely many arbitrarily large integers $T \in h(h+1) d \mathbf{N}$ so that

$$
\begin{aligned}
& \left\{\frac{T \theta_{j}}{2 \pi}\right\}>1-\epsilon>1-\frac{1}{2 k}, \quad \text { for } j \in P, \\
& \left\{\frac{T \theta_{j}}{2 \pi}\right\}<\epsilon<\frac{1}{2 k}, \quad \text { for } j \in\{1, \ldots, k\} \backslash P .
\end{aligned}
$$

By (3.7), the choice of $T$, and the irrationality of all $\frac{T \theta_{j}}{2 \pi}$ 's, $1 \leq j \leq k$, we get

$$
\begin{aligned}
0<2 \sum_{i=1}^{k}\left\{\frac{T \theta_{i}}{2 \pi}\right\} & =\frac{2(d h+d-2) T}{h(h+1) d}-(d-1-k) T-2 \sum_{i=1}^{k}\left[\frac{T \theta_{i}}{2 \pi}\right] \\
& \in \mathbf{N} \cap[1,2 k] .
\end{aligned}
$$

On the other hand, by (3.9) and (3.10) it yields $A\left(1-\frac{1}{2 k}\right)<$ $\sum_{i=1}^{A}\left\{\frac{T \theta_{i}}{2 \pi}\right\}<A$ and $0<\sum_{i=A+1}^{k}\left\{\frac{T \theta_{i}}{2 \pi}\right\}<\frac{k-A}{2 k}$. Thus we obtain

$$
\begin{aligned}
2 \sum_{i=1}^{k}\left\{\frac{T \theta_{i}}{2 \pi}\right\} & =2 \sum_{i=1}^{A}\left\{\frac{T \theta_{i}}{2 \pi}\right\}+2 \sum_{i=A+1}^{k}\left\{\frac{T \theta_{i}}{2 \pi}\right\} \\
& \in\left(2 A-\frac{A}{k}, 2 A+1-\frac{A}{k}\right) \subset(2 A-1,2 A+1) .
\end{aligned}
$$

Thus by (3.11) and (3.12) it yields $2 \sum_{i=1}^{k}\left\{\frac{T \theta_{i}}{2 \pi}\right\}=2 A$. Then by (3.5) and (3.7) we obtain

$$
\begin{aligned}
i\left(c^{T}\right) & =(d-1-k) T+2 \sum_{i=1}^{k}\left[\frac{T \theta_{i}}{2 \pi}\right]+k \\
& =(d-1-k) T+2 \sum_{i=1}^{k} \frac{T \theta_{i}}{2 \pi}-2 \sum_{i=1}^{k}\left\{\frac{T \theta_{i}}{2 \pi}\right\}+k \\
& =\frac{2(d h+d-2) T}{h(h+1) d}-2 A+k .
\end{aligned}
$$

This completes the proof of Claim 1.

Then Theorem 2.6 and Claim 1 yield

$$
\begin{aligned}
i\left(c^{m}\right) \geq & i\left(c^{T}\right)+i(c)+2 A-k \\
& =\frac{2(d h+d-2) T}{h(h+1) d}+(d-1), \quad \forall m \geq T+1, \\
i\left(c^{m}\right) \leq & i\left(c^{T}\right)-i(c)+2 A-k \\
& =\frac{2(d h+d-2) T}{h(h+1) d}-(d-1), \quad \forall 1 \leq m \leq T-1 .
\end{aligned}
$$


Set $\hat{T}=\frac{2(d h+d-2) T}{h(h+1) d}$. Then $\hat{T} \in 2 \mathbf{N}$ may be arbitrarily large by the choice of $T$ in Claim 1, and then $\hat{T}-(d-1)$ is odd.

Now by (3.14) and Proposition 2.1, all iterates $c^{m}$ with $m \geq T+1$ have no contribution to the sum $\sum_{q=0}^{\hat{T}-(d-1)} m_{q}$. On the other hand, note that all iterated indices in Case 1 are odd. So by (3.15) and Proposition 2.1, each iterate $c^{m}$ with $1 \leq m \leq T-1$ contributes precisely 1 to the sum $\sum_{q=0}^{\hat{T}-(d-1)} m_{q}$. Finally, by Claim 1, it is possible that the iterate $c^{T}$ contributes either 1 or 0 to this sum depending on whether the value of $i\left(c^{T}\right)$ is less than or equal to $\hat{T}-(d-1)$. Therefore, in Case 1 we have

$$
\sum_{q=0}^{\hat{T}-(d-1)} m_{q} \in\{T, T-1\} .
$$

On the other hand, by (2.7) of Lemma 2.4 with even $d \geq 2$ and $D=d(h+1)-2$, we obtain

$$
\sum_{q=0}^{\hat{T}-(d-1)} b_{q}<h\left(\frac{D}{2}+1\right) \frac{\hat{T}-2(d-1)}{D}-\frac{h(h-1) d}{4}+2 .
$$

Therefore, by (3.2), (3.16), and (3.17) we have

$$
\begin{aligned}
T-1 & \leq \sum_{q=0}^{\hat{T}-(d-1)} m_{q}=\sum_{q=0}^{\hat{T}-(d-1)} b_{q} \\
& <h\left(\frac{D}{2}+1\right) \frac{\hat{T}-2(d-1)}{D}-\frac{h(h-1) d}{4}+2 \\
& =\frac{h(D+2)}{2 D} \hat{T}-\frac{h(D+2)(d-1)}{D}-\frac{h(h-1) d}{4}+2 \\
& =T-h(d-1)-\frac{2 h(d-1)}{D}-\frac{(h-1) h d}{4}+2 .
\end{aligned}
$$

Notice that $h \geq 2, d \geq 2$, and $D=d(h+1)-2>0$. So we have

$$
h(d-1) \geq 2, \quad \frac{2 h(d-1)}{D}>0, \quad \frac{(h-1) h d}{4} \geq 1 .
$$

Now we obtain a contradiction by (3.18) and (3.19), and this completes the proof of Theorem 1.1 in Case 1.

Case 2. $k=d h-1-\left(h_{-}+h_{+}+2 k_{*}+2 k_{0}\right) \in 2 \mathbf{N}$.

In this case, $i\left(c^{m}\right)-i(c)$ is odd for $m \in 2 \mathbf{N}$ and is even for $m \in 2 \mathbf{N}-1$ by (3.5) and $i(c)=d-1 \in 2 \mathbf{N}-1$. Thus by Definition 2.2 we get $\gamma_{c}=-\frac{1}{2}$, which, together with Proposition 2.3, yields

$$
-\frac{1}{2 \hat{i}(c)}=B(d, h)=-\frac{h(h+1) d}{2 d(h+1)-4} .
$$


Thus by (3.5) we obtain

$$
d-1-k+\sum_{i=1}^{k} \frac{\theta_{i}}{\pi}=\hat{i}(c)=\frac{d(h+1)-2}{h(h+1) d} .
$$

So in this case, we have

$$
\begin{aligned}
i(c) & =d-1, \\
m \hat{i}(c) & =(d-1-k) m+2 \sum_{i=1}^{k} \frac{m \theta_{i}}{2 \pi} \\
& =\frac{(d h+d-2) m}{h(h+1) d}, \quad \forall m \geq 1 .
\end{aligned}
$$

Claim 2. There exist an integer $A$ with $\left[\frac{k+1}{2}\right] \leq A \leq k$ and a subset $P$ of integers $\{1, \ldots, k\}$ with $A$ integers such that there exist infinitely many arbitrarily large integers $T \in 2 h(h+1) d \mathbf{N}$ satisfying

$$
i\left(c^{T}\right)=\frac{(d h+d-2) T}{h(h+1) d}-(2 A-k) .
$$

In fact, by the same arguments in the proof of Claim 1, we get $2 \sum_{i=1}^{k}\left\{\frac{T \theta_{i}}{2 \pi}\right\}=2 A$, too. Therefore (similarly to (3.13)), by (3.5), (3.7), and (3.21), Claim 2 holds.

Then Theorem 2.6 and Claim 2 yield

$$
\begin{aligned}
i\left(c^{m}\right) \geq & i\left(c^{T}\right)+i(c)+2 A-k \\
& =\frac{(d h+d-2) T}{h(h+1) d}+(d-1), \quad \forall m \geq T+1, \\
i\left(c^{m}\right) \leq & i\left(c^{T}\right)-i(c)+2 A-k \\
& =\frac{(d h+d-2) T}{h(h+1) d}-(d-1), \quad \forall 1 \leq m \leq T-1 .
\end{aligned}
$$

Set $\hat{T}=\frac{(d h+d-2) T}{h(h+1) d}$. Then we have $\hat{T} \in 2 \mathrm{~N}, \hat{T}-(d-1)$ is odd, and $\hat{T}-(d-1) \geq h d-1$. By $(3.23)$ and Proposition 2.1, all iterates $c^{m}$ with $m \geq T+1$ have no contribution to the sum $\sum_{q=0}^{\hat{T}-(d-1)} m_{q}$. On the other hand, in Case 2 by (3.5) the index $i\left(c^{m}\right)$ and $m$ have the same parity. Thus all the iterates $c^{m}$ with even $m \in \mathbf{N}$ including $c^{T}$ contribute nothing to the sum $\sum_{q=0}^{\hat{T}-(d-1)} m_{q}$ by the definition (3.1) and Claim 2 . Only the iterates $c^{m}$ with odd $m$ can contribute to this sum. By (3.23), the iterates $c^{m}$ contribute nothing to this sum when $m \geq T+1$. By (3.24), each $c^{m}$ with odd $m \leq T-1$ contributes precisely 1 to this sum. Therefore, we obtain

$$
\sum_{q=0}^{\hat{T}-(d-1)} m_{q}=\frac{T}{2}
$$


Then, by (3.2), (3.25), and (2.7) of Lemma 2.4 with even $d \geq 2$ and $D=d(h+1)-2$, we obtain

$$
\begin{aligned}
\frac{T}{2} & =\sum_{q=0}^{\hat{T}-(d-1)} m_{q}=\sum_{q=0}^{\hat{T}-(d-1)} b_{q} \\
& <h\left(\frac{D}{2}+1\right) \frac{\hat{T}-2(d-1)}{D}-\frac{h(h-1) d}{4}+2 \\
& =\frac{h(D+2)}{2 D} \hat{T}-\frac{h(D+2)(d-1)}{D}-\frac{h(h-1) d}{4}+2 \\
& =\frac{T}{2}-h(d-1)-\frac{2 h(d-1)}{D}-\frac{(h-1) h d}{4}+2 .
\end{aligned}
$$

Notice that $h \geq 2, d \geq 2$, and $D=d(h+1)-2>0$. So we have

$$
h(d-1) \geq 2, \quad \frac{2 h(d-1)}{D}>0, \quad \frac{(h-1) h d}{4} \geq 1 .
$$

Then we obtain a contradiction from (3.26) and complete the proof of Theorem 1.1 in Case 2.

Case 3. $k=0$.

In this case, by $(3.5)$ we have $i\left(c^{m}\right)=m(d-1)$ for all $m \geq 1$. In other words, the iterated Morse indices of the closed geodesic $c$ are strictly monotone increasing in the iteration time $m$. So it follows from Proposition 2.1 that $M_{q} \leq 1$ for every odd $q \in \Omega(d, h)$ defined by $(2.5)$. On the other hand, by (2.6) it yields $b_{q}=h+1 \geq 3$ for every odd $q \in \Omega(d, h)$ defined by $(2.5)$. Then by Lemma 3.2 we obtain $1 \geq M_{q}=$ $b_{q}=h+1 \geq 3$ for such odd $q$ just mentioned above. This contradiction completes the proof for Case 3 .

Now Theorem 1.1 follows from the proofs in Cases 1-3. $\quad$ q.e.d.

\section{References}

[Ano] D.V. Anosov, Geodesics in Finsler geometry, Proc. I.C.M. (Vancouver, B.C. 1974), Vol. 2, 293-297, Montreal, 1975 [Russian]; Amer. Math. Soc. Transl. 109 (1977) 81-85, MR 0426058, Zbl 0368.53045.

[Ban] V. Bangert, Geodätische Linien auf Riemannschen Mannigfaltigkeiten, Jber. Deutsch. Math.-Verein 87 (1985), 39-66, MR 0789708, Zbl 0565.53028.

[BaL] V. Bangert \& Y. Long, The existence of two closed geodesics on every Finsler n-sphere, Math. Ann. 346 (2010), 335-366, MR 2563691, Zbl 1187.53040.

[DuL1] H. Duan \& Y. Long, Multiple closed geodesics on bumpy Finsler $n$-spheres, J. Diff. Equa. 233 (2007), 221-240, MR 2290278, Zbl 1106.53048.

[DuL2] H. Duan \& Y. Long, The index growth and multiplicity of closed geodesics, J. Funct. Anal. 259 (2010), 1850-1913, MR 2665413, Zbl 1206.53043.

[Fet] A. Fet, A periodic problem in the calculus of variations, Dokl. Akad. Nauk. SSSR (N.S.) 160 (1965), 287-289 [Russian]; Soviet Math. 6 (1965), 85-88, MR 0220316, Zbl 0135.32604. 
[GrM] D. Gromoll \& W. Meyer, Periodic geodesics on compact Riemannian manifolds, J. Diff. Geom. 3 (1969), 493-510, MR 026455, Zbl 0203.54401.

[Hin] N. Hingston, Equivariant Morse theory and closed geodesics, J. Diff. Geom. 19 (1984), 85-116, MR 0739783, Zbl 0561.58007.

[HiR] N. Hingston \& H.-B. Rademacher, Resonance for loop homology of spheres, J. Diff. Geom. 93 (2013), 133-174, MR 3019513, Zbl 1285.53031.

[Jos] J. Jost, Riemannian Geometry and Geometric Analysis, Springer, Heldeburg, 2011, MR 2829653, Zbl 1227.53001.

[Kat] A.B. Katok, Ergodic properties of degenerate integrable Hamiltonian systems, Izv. Akad. Nauk SSSR 37 (1973) [Russian]; Math. USSR-Izv. 7 (1973), 535-571, MR 0331425, Zbl 0316.58010.

[Kli] W. Klingenberg, Lectures on Closed Geodesics, Springer, Berlin, 1978, MR 0478069, Zbl 0397.58018.

[Liu] C. Liu, The relation of the Morse index of closed geodesics with the Maslovtype index of symplectic paths, Acta Math. Sinica (English Series) 21 (2005), 237-248, MR 2135288, Zbl 1092.58009.

[LiL] C. Liu \& Y. Long, Iterated index formulae for closed geodesics with applications, Science in China 45 (2002), 9-28, MR 1894955, Zbl 1054.53063.

[Lon1] Y. Long, Bott formula of the Maslov-type index theory, Pacific J. Math. 187 (1999), 113-149, MR 1674313, Zbl 0924.58024.

[Lon2] Y. Long, Precise iteration formulae of the Maslov-type index theory and ellipticity of closed characteristics, Adv. in Math. 154 (2000), 76-131, MR 1780096, Zbl 0970.37013.

[Lon3] Y. Long, Index Theory for Symplectic Paths with Applications, Progress in Math. 207, Birkhäuser, Basel, 2002, MR 1898560, Zbl 1012.37012.

[Lon4] Y. Long, Multiplicity and stability of closed geodesics on Finsler 2-spheres, J. of Euro. Math. Soc. 8 (2006), 341-353, MR 2239281, Zbl 1099.53036.

[LoD] Y. Long \& H. Duan, Multiple closed geodesics on 3-spheres, Adv. Math. 221 (2009), 1757-1803, MR 2522828, Zbl 1172.53027.

[LoW] Y. Long \& W. Wang, Stability of closed geodesics on Finsler 2-spheres, J. Funct. Anal. 255 (2008), 620-641, MR 2426431, Zbl 1151.53037.

[LoZ] Y. Long \& C. Zhu, Closed characteristics on compact convex hypersurfaces in $\mathbf{R}^{2 n}$, Ann. of Math. 155 (2002), 317-368, MR 1906590, Zbl 1028.53003.

$[\mathrm{LyF}]$ L.A. Lyusternik \& A.I. Fet, Variational problems on closed manifolds, Dokl. Akad. Nauk SSSR (N.S.) 81 (1951), 17-18 [Russian], MR 0044760, Zbl 0045.20903.

[Rad1] H.-B. Rademacher, On the average indices of closed geodesics, J. Diff. Geom. 29 (1989), 65-83, MR 0978076, Zbl 0658.53042.

[Rad2] H.-B. Rademacher, Morse Theorie und geschlossene Geodatische, Bonner Math. Schriften 229 (1992), MR 1237191, Zbl 0826.58012.

[Rad3] H.-B. Rademacher, Existence of closed geodesics on positively curved Finsler manifolds, Ergodic Theory Dynam. Systems 27 (2007), 957-969, MR 2322187, Zbl 1124.53018.

[Rad4] H.-B. Rademacher, The second closed geodesic on Finsler spheres of dimension $n>2$, Trans. Amer. Math. Soc. 362 (2010), 1413-1421, MR 2563734, Zbl 1189.53039. 
[Rad5] H.-B. Rademacher, The second closed geodesic on the complex projective plane, Front. Math. China 3 (2008), 253-258, MR 2395220, Zbl 1148.53028.

[She] Z. Shen, Lectures on Finsler Geometry, World Scientific, Singapore, 2001, MR 1845637, Zbl 0974.53002.

[ViS] M. Vigué-Poirrier \& D. Sullivan, The homology theory of the closed geodesic problem, J. Diff. Geom. 11 (1976), 633-644, MR 0455028, Zbl 0361.53058.

[Wan] W. Wang, Closed geodesics on positively curved Finsler spheres, Adv. Math. 218 (2008), 1566-1603, MR2419933, Zbl 1148.53029.

School of Mathematical Sciences and LPMC NANKAI UNIVERSITY

Tianjin 300071, The People's Republic of China E-mail address: duanhg@nankai.edu.cn

Chern Institute of Mathematics and LPMC NANKAI UNIVERSity

Tianjin 300071, The People's Republic of China E-mail address: longym@nankai.edu.cn

School of Mathematical Sciences and LMAM Peking University Beijing, 100871, The People's Republic of China E-mail address: alexanderweiwang@gmail.com 